\title{
The role of task difficulty in a discrete tracking task
}

DANIEL L. BATY

AMES RESEARCH CENTER, NASA

Six Ss performed a self-paced, random-input, discrete tracking task in one and two dimensions by quickly touching welldefined lighted areas with a stylus. An index of task difficulty was computed for each of 14 experimental conditions. Performance in terms of information-processing rate was shown to be primarily determined by the degree of task difficulty and the number of target alternatives.

In an earlier study, Klemmer (1956) computed the information-processing rate $\left(I_{R}\right.$ in bits/sec.) for $S s$ performing a discrete tracking task in one and two dimensions, a task similar to the one to be described for the present experiment. In Klemmer's experiment and the experiments to be described, the information value of each stimulus presentation $\left(\mathrm{I}_{V}\right)$ is $\log _{2} \mathrm{~N}$, where $\mathrm{N}$ is the number of stimulus alternatives; $I_{R}$ is the average rate of processing the presented information. Klemmer presented four different stimulus conditions $\left(I_{V}=2,3,4\right.$, and 5 bits/presentation) as one-dimensional (1-D) tasks, and four different stimulus conditions $\left(\mathrm{I}_{\mathrm{V}}=4,6,8\right.$, and $10 \mathrm{bits} / \mathrm{presen}-$ tation) as two-dimensional (2-D) tasks. He found that $I_{R}$ increased as $I_{V}$ increased, but at a decreasing rate, so that performance approached horizontal asymptotes of approximately $4.2 \mathrm{bits} / \mathrm{sec}$. for 1-D and $6.6 \mathrm{bits} / \mathrm{sec}$. for 2-D. Klemmer concluded that these values were near the maximum informationprocessing rate for this type of task. It was implied that the difference in asymptotic levels for the 1-D and 2-D tasks was due to the difference in dimensionality.

Fitts \& Peterson (1964) defined an index of task difficulty (ID) for a single reaction motor task as ID $=\log _{2}(2 \mathrm{~A} / \mathrm{W})$, where $\mathrm{A}$ was the distance of hand travel and $W$ the width of the target to be hit. Since all conditions were two-choice tasks, only one moving distance had to be measured for any one part of the experiment, and $I_{V}$ was always equal to 1 . An estimate of $I_{R}$ for each condition showed that $I_{R}$ was a steadily decreasing function of $\mathrm{D}$, a result which suggested that an index of difficulty may also have been an important variable in a study such as Klemmer's. For this experiment, Fitts and Peterson's definition of ID was adapted for tasks with higher values of $I_{V}$ by substituting average hand-travel distance during a sequence of responses $\bar{A}$ for the single distance $A$ to obtain $\bar{D}$, i.e., average ID.

Since Klemmer's inputs were randomly selected with equal probabilities, and the dimensions for each task were given, it was possible to estimate $\overline{\mathrm{ID}}$ for each of his conditions. In Fig. 1 , his values of $I_{R}$ are plotted against $\overline{\mathrm{ID}}$. Note that only two points in Klemmer's experiment had equal values of $\mathrm{I}_{\mathrm{V}}$, points $K: 16(1-D)$ and $K: 4 \times 4 \quad(2-D)$ with $I_{V}=4$. It is clear that the absolute value of $I_{R}$ was greater for the 2-D task than for the 1-D task (distance V). But, as shown in Fig. 1, $\overline{\mathrm{ID}}$ is less for the 2-D task than for the 1-D task. It is not clear whether $I_{R}$ is greater for condition $K: 4 \times 4$ because of the different dimensionality or because of the lower $\overline{\mathrm{ID}}$. The present experiment was designed to measure $I_{R}$ for $1-D$ and 2-D tasks with matched values of $I_{V}$ and $\overline{I D}$.

\section{Method}

A 12.5-in.-square tracking surface was divided into a 25 by 25 matrix of 0.5 -in.-square cells, each deep enough to hold one small neon bulb below a glass cover. All cells were covered with a sheet of frosted mylar so that a light within a cell resulted in a homogeneously lighted 0.5-in.-square surface. For each of the 14 experimental conditions, an opaque mask with either four or 16 square holes (i.e., $\mathrm{IV}_{\mathrm{V}}=$ 2 or 4 bits/presentation) was placed over the mylar and under the glass, making the number and location of the stimulus choices explicit. Each mask had a

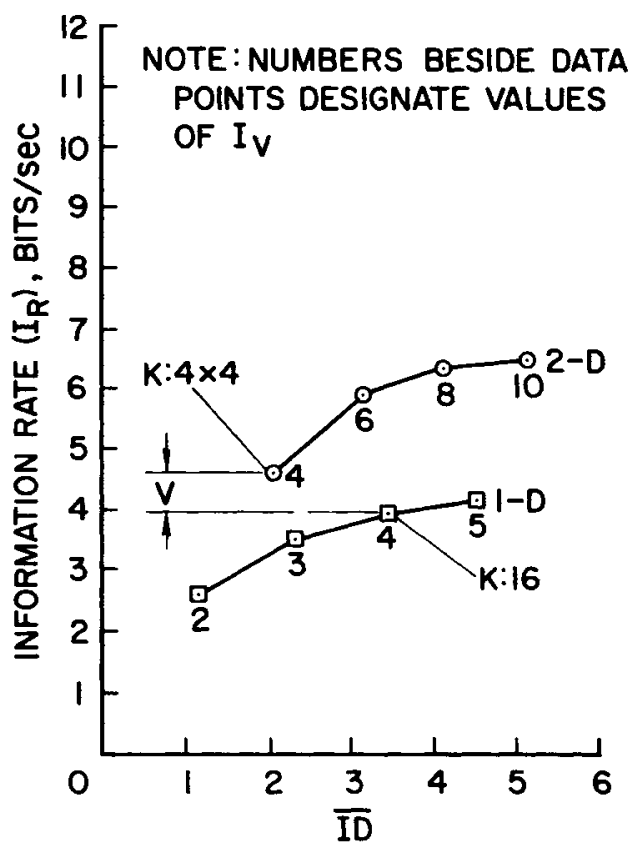

Fig. 1. Results of Klemmer's (1956) experiment plotted against $\overline{\mathbf{I D}}$ 
different combination of hole size and spacing corresponding to a given value of $\overline{\mathbf{D}}$. Two of the conditions were designed to have exactly the same task dimenslons as Klemmer's $\mathrm{K}: 16$ and $\mathrm{K}: 4 \times 4$. $\overline{\mathrm{ID}}$ was defined as $\log _{2}(2 \bar{A} / W)$, where $W$ was the width of the target and $\vec{A}$ the average movement distance of the stylus tip for each response during the run.

Six male engineers and scientists, 28 to 42 years of age and free from obvious physical defects, volunteered as Ss. Their task was to touch each lighted area with a stylus as quickly as possible after the onset of the light. The test equipment was designed so that the touched light went out immediately and another randomly chosen (tape-controlled) light came on within 7 msec. Each run continued for 105 stimulus presentations. A timer automatically starter after the fifth response so that all times were recorded for 100 responses. The stylus tip contained a photocell. A correct response was registered when there was a combination of photocell response and stylus contact with the board. To prevent a "sliding" strategy while going from one lighted area to another (when not adjacent), an error was counted each time the stylus made contact outside a lighted area. Ten errors were allowed per run before the run was automatically stopped and then restarted.

The experiment was conducted in a dimly lighted room to prevent spurious photocell responses. $S$ sat on a high stool so that his line of sight was approximately perpendicular to the tracking surface. No knowledge of results was given during the experiment. Each session consisted of one run on each of the 14 conditions, and lasted approximately $40 \mathrm{~min}$. There were three practice sessions and nine data sessions. Nine different input tapes were used for the data sessions to prevent any chance of Ss memorizing partial input sequences. The sequence of conditions within a tape was randomly chosen as was the sequence of assigning the tapes to Ss. Within each run the stimuli were presented randomly with equal probability with replacement; i.e., a given light could repeat one or more times as the stimulus. If a light repeated immediately, contact with the board had to be broken and the light touched again. The primary instruction to $S$ was to proceed as rapidly as possible after starting the run.

\section{Results and Discussion}

The average performance of six Ss for nine experimental sessions is shown in Fig. 2. For both values of $I_{V}, I_{R}$ was slightly greater for the 1-D tasks than for the 2-D tasks. In this experiment the average standard deviation of the run times was $3.42 \%$ of the total mean time per run, suggesting that the overall performance was consistent.

In Fig. 2 a perpendicular line through $\mathrm{K}: 16$ inter-

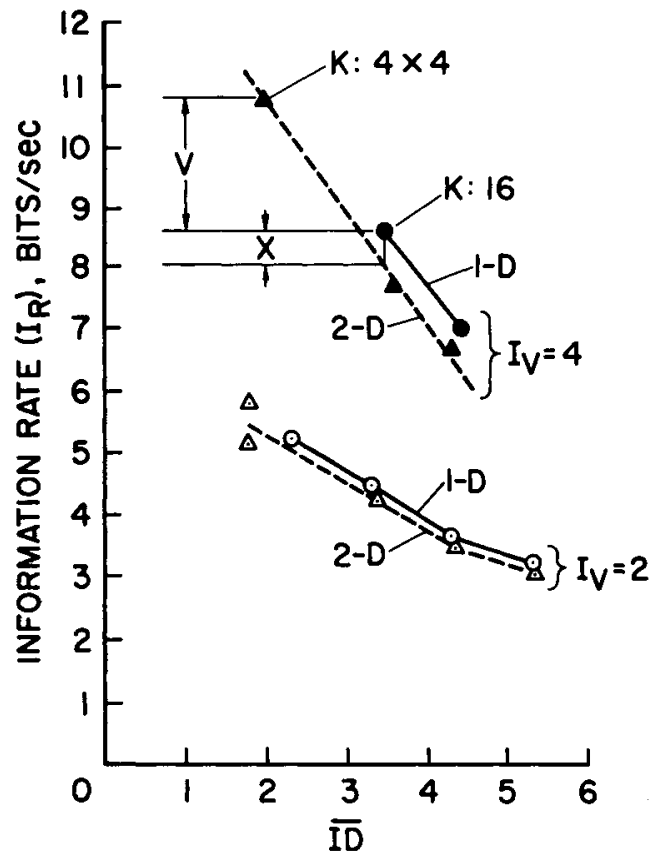

Fig. 2. The effect of $\overline{\mathrm{ID}}$ on $\mathrm{I}_{\mathbf{R}}$; mean rates from six $\mathrm{Ss}$.

sects the fitted 2-D line $\left(I_{V}=4\right)$ at the value of $I_{R}$ that is the best estimate of performance for a 2-D task with the same value of $\overline{D D}$ as $K: 16$. This difference, $0.45 \mathrm{bits} / \mathrm{sec}$., distance $\mathrm{X}$, is to be compared with distance V, $2.2 \mathrm{bits} / \mathrm{sec}$, , which is the absolute difference in performance on the two tasks that were geometrically the same as Klemmer's K:16 and K:4 x 4 . Based on these data, approximately $80 \%$ of the difference in performance for the two conditions is due to the difference in $\overline{\mathrm{ID}}$ and the remainder is due to dimensionality factors.

The difference in rates between the two studies is explained by the fact that Klemmer's Ss were required to "press down slightly," whereas for this study a slight contact closed an electronic circuit, allowing a quick touch-and-go strategy.

For this type of experiment, the main advantage of the 2-D task over the 1-D task, if target sizes are held constant, is that IV can be made much larger for the 2-D task without increasing the values of $\overline{\mathrm{ID}}$. Thus, the higher information-processing rates possible for 2-D tasks are directly attributable to lower values of $\overline{\mathrm{ID}}$.

\section{References}

Fitts, P. M., \& Peterson, J. R. Information capacity of discrete motor responses. J. exp. Psychol., 1964, 67, 103-112.

Klemmer, E. T. Discrete tracking in one and two dimensions. Operational Applications Laboratory, Air Force Cambridge Research Center, USAF Rep. AFCRC-TN-56-2, 1956. 